\title{
Mikrooptischer Sensor für die Volumenstrommessung in Kapillaren
}

\author{
Ralf Müller, Arndt Steinke \\ CiS Forschungsinstitut für Mikrosensorik und Photovoltaik GmbH, Erfurt, Konrad-Zuse-Straße 14
}

\section{Zusammenfassung}

In elektrochemischen Anlagen für die Erzeugung von metallisierten Mikrostrukturen ist es wichtig, den konvektiven Stoffaustausch in diesen Mikrostrukturen (z. B. Bohrungen) zu kennen. In dieser Arbeit wird ein erstes Funktionsmuster eines ,faserfreien“ integriert optischen Durchflussmesser auf Basis der Ortsfiltertechnik, der bei frei angeströmten Mikrostrukturen in prozesstechnischen Anlagen eingesetzt werden kann, vorgestellt. Grundlage für die Funktion ist das Vorhandensein von Tracerpartikeln. Es werden u. a. ein (Vertical Cavity Surface Emitting Laser \{VCSEL\}), eine $2 \mathrm{~mm}$ lange Kapillare (Innendurchmesser: 0,15 mm; Außendurchmesser: $5 \mathrm{~mm}$ ) und ein spezieller Siliziumchip, der am CiS Forschungsinstitut hergestellt wird, eingesetzt. Für die Aufnahme von ersten Messergebnissen wurden als Tracerpartikel Hohlglaskugeln mit einem Durchmesser von $10 \mu \mathrm{m}$ verwendet. Diese wurden in deionisiertem Wasser dispergiert.

\section{Einleitung}

Neu zu entwickelnde elektrochemische Anlagen für die Erzeugung von metallisierten Mikrostrukturen in vergleichsweise großen Oberflächen müssen hydrodynamisch optimiert werden, damit während des gesamten Prozesses der konvektive Stoffaustausch in den Mikrostrukturen ein für jeden Anwendungsfall abschätzbares unteres Limit nicht unterschreitet und gleichförmige Schichtdicken resultieren. Ein wesentliches Problem für Anlagenentwickler und -betreiber ergibt sich daraus, dass bisher nur sehr eingeschränkt Methoden verfügbar sind, die Strömungsmessungen in frei angeströmten Mikrostrukturen ermöglichen, die sich in komplexen prozesstechnischen Anlagen mit eingeschränkter optischer Zugänglichkeit befinden. Sehr eingeschränkt bedeutet, dass bisher lediglich eine faseroptische Lösung in einer halbtechnischen Anlage, die mit Wasser arbeitet, getestet wurde [1].

Das Ziel der Untersuchungen hier besteht darin, einen „faserfreien“ integriert optischen Durchflussmesser auf Basis der Ortsfiltertechnik, der bei frei angeströmten Mikrostrukturen in prozesstechnischen Anlagen eingesetzt werden kann, zu realisieren. Grundlage dafür ist das Vorhandensein von Tracerpartikeln.

\section{Messprinzip}

Die Bestimmung des Volumenstroms $q_{\mathrm{v}}$ nutzt das für laminare Rohrströmungen Newtonscher Flüssigkeiten bekannte Ergebnis, nach dem die Geschwindigkeit $U_{\max }$ in der Rohrmitte gleich der doppelten mittleren Geschwindigkeit $u_{\mathrm{m}}$ ist. Bei bekannter Rohrquerschnittsfläche ist der Volumenstrom gleich dem Produkt aus mittlerer Geschwindigkeit $u_{\mathrm{m}}$ und Rohrquerschnittsfläche. Voraussetzung dabei ist die vollständige Ausbildung des laminaren Strömungsprofils.

$U_{\max }$ wird mit einem optoelektronischen Ortsfrequenzfilter (Ortsfilter) bestimmt. In der Flüssigkeit sind Tracerpartikel enthalten, deren Geschwindigkeiten $U$ mit Hilfe dieses optischen Ortsfilters gemessen werden. Dazu werden die Tracerpartikel mit einem Laser beleuchtet, so dass der Partikelschatten auf den optischen Ortsfilter fällt. Das unmittelbar an der Außenwand der Kapillare angeordnete Ortsfilter ist ein differentielles Ortsfilter, das aus zwei identischen Ortsfiltern besteht, die um die halbe Ortsfilterkonstante $g$ verschoben sind.

Die Geschwindigkeiten $U$ und damit auch $U_{\max }$ ergibt sich aus: 


$$
U=\frac{g}{T},
$$

wobei $T$ die Periodendauer für das Differenzsignal der beiden Ortsfilter beim Partikeldurchgang ist.

\section{Aufbau des Sensors}

Für den Sensor wurde ein spezieller Siliziumchip entwickelt (vgl. Bild 1).

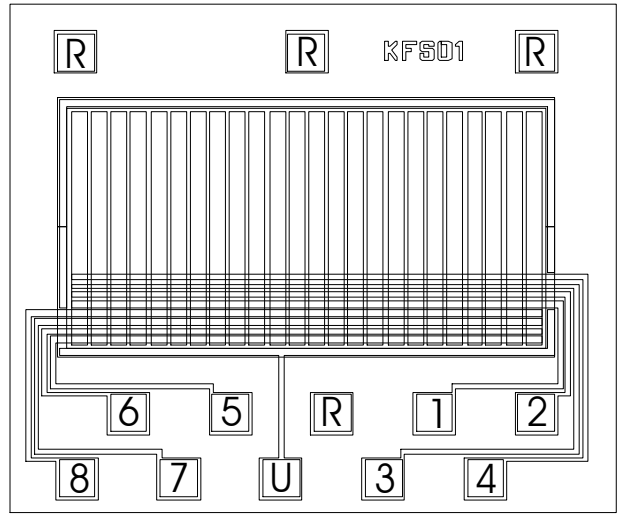

Bild 1 Layout für das entwickelte Silizium-Fotodiodenchip, mit der Möglichkeit die Gitterkonstante umzuschalten, 1...8 Anodenkontakte, R Rückseitenkontakte.

Auf dem Chip befinden sich 24 Fotodiodenstreifen mit je einer Breite von $50 \mu \mathrm{m}$. Basis für den mikrooptischen Sensor ist ein Grundglasträger von $10 \times 20 \mathrm{~mm}^{2}$, der auch die Signalvorverarbeitung tragen wird. Ein Verdrahtungsträger, der ebenfalls aus Glas beschaffen ist und den Si-Chip trägt (Flip-Chip), steht senkrecht auf dem Grundglasträger. Die Verdrahtung auf dem Glas wurde mittels eines Schichtsystems aus CrNi-Al-FeNi-Au realisiert. Bild 2 b) zeigt den Konstruktionsansatz grafisch und Bild 2 a) das Ergebnis einer Berechnung mit dem Prinzip der Strahlenverfolgung.

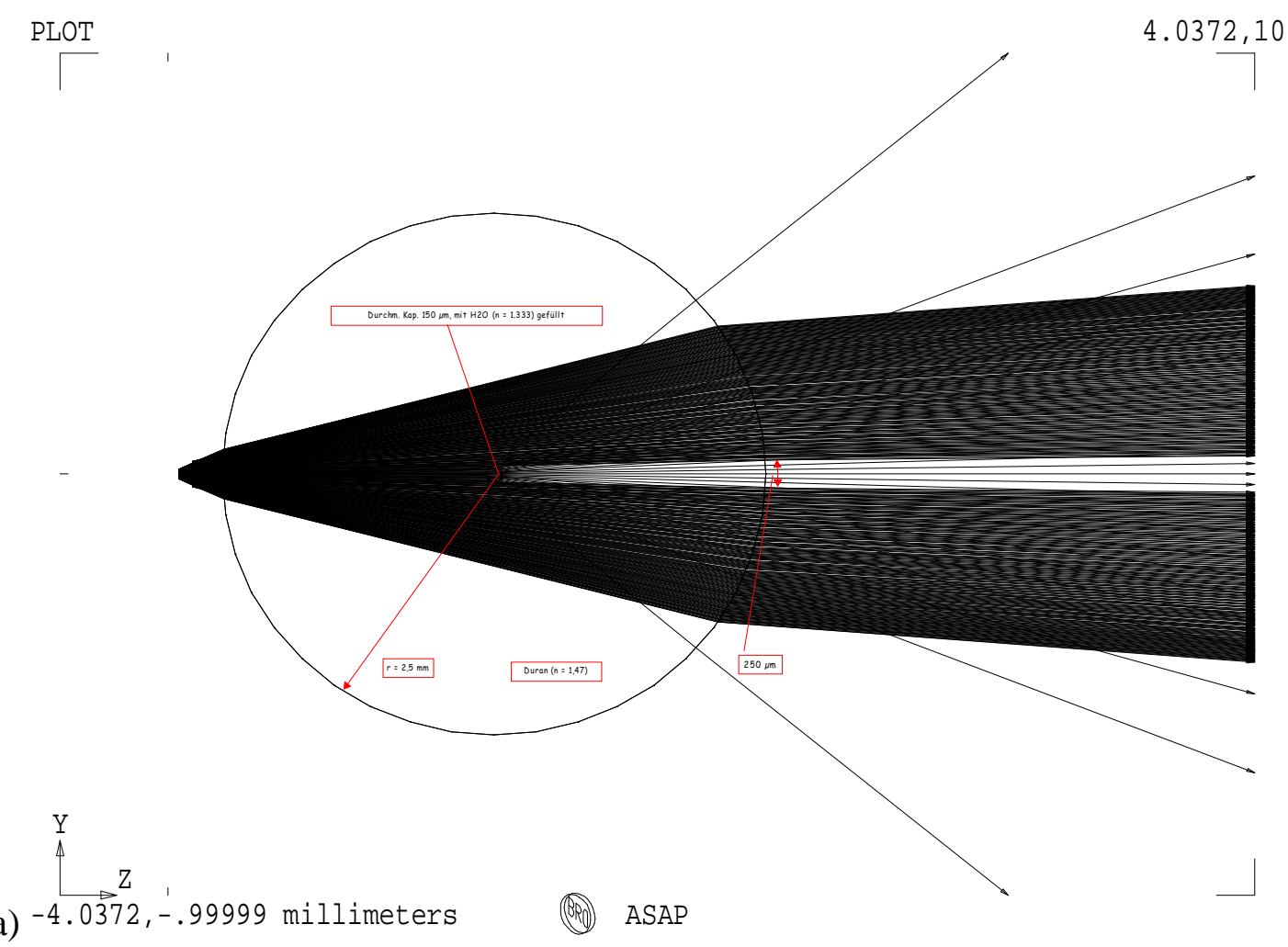




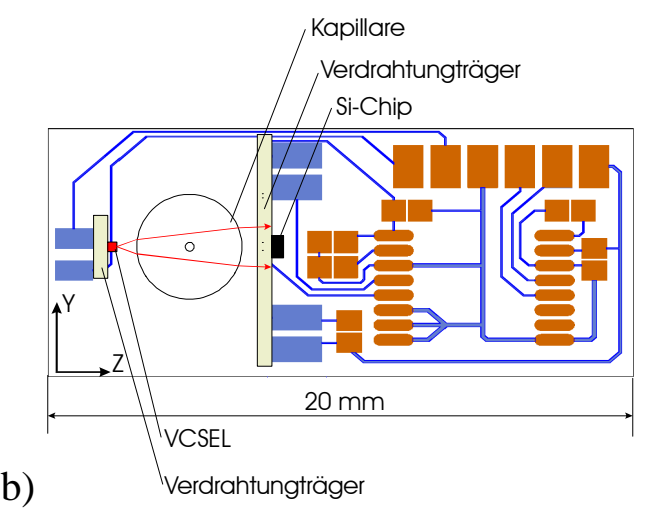

Bild 2 Konzeption für den Sensor zur freien Anströmung, Blick von oben, Dicke des Moduls in X-Richtung: 2,5 mm (links); Ergebnis für die Berechung mit dem Prinzip der Strahlenverfolgung (rechts).

Bild 2 (rechts) zeigt, dass die aktive Chipfläche innerhalb eines Lichtspalts, wo die Modulationstiefe maximal ist, zu positionieren ist. Dies erfordert eine hohe Montagegenauigkeit.

In Bild 3 ist ein erstes Funktionsmuster dargestellt. Die Signalvorverarbeitung ist hier noch nicht mit implementiert, es wurde für die Zugentlastung der Anschlussleitung eine weitere Glasplatte angeklebt und der abschließende Verguss mit einem optischen Epoxidharz steht noch aus.

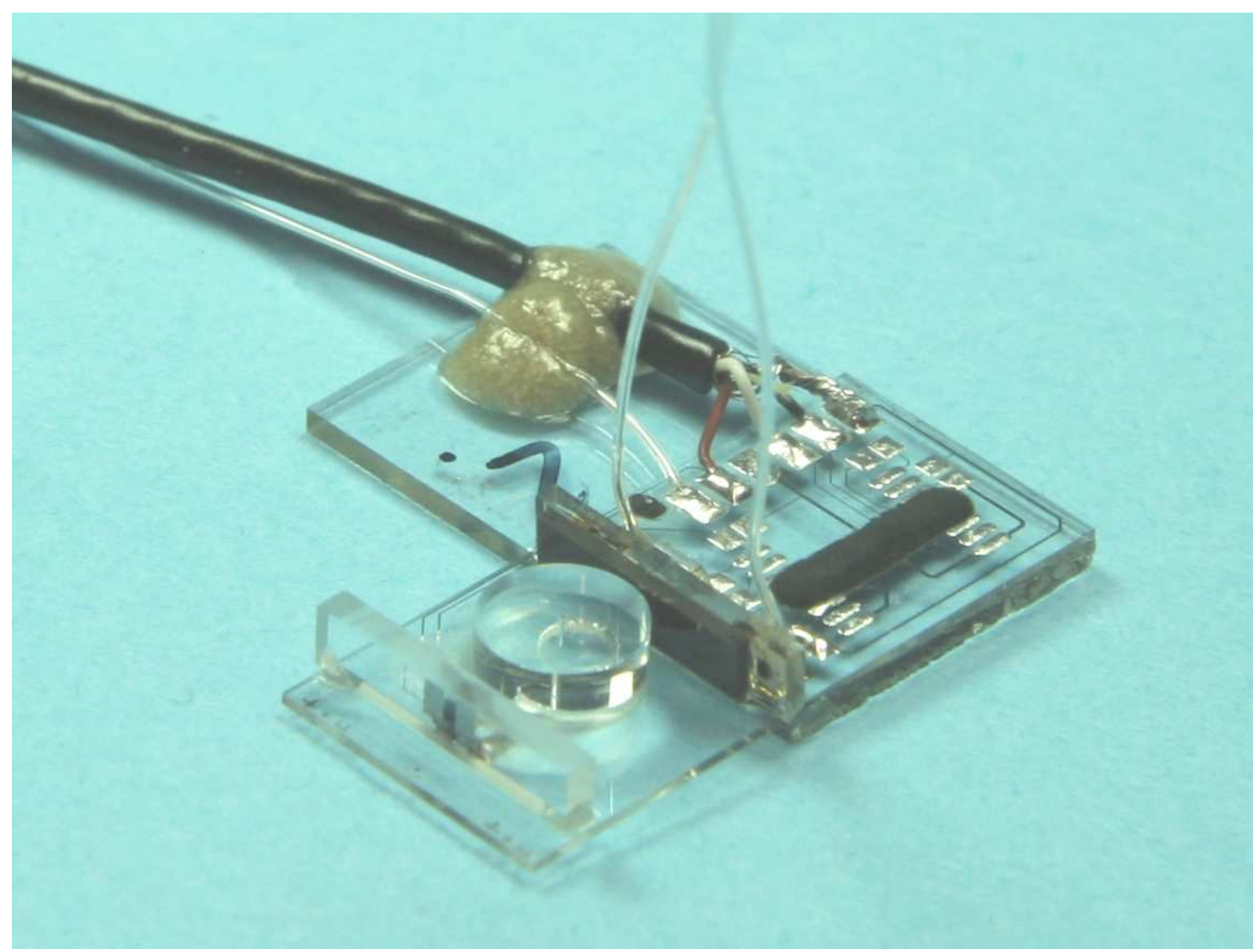

Bild 3 Fotografische Darstellung eines ersten Funktionsmusters. 


\section{Messergebnisse}

In Vorversuchen wurde der Aufbau mit einer $20 \mathrm{~cm}$ langen Kapillare realisiert. Mittels einer Infusionspumpe (Perfusor compact S, Fa. B. Braun) wurde der Volumenstrom eingestellt. Als Messmedium wurde deionisiertes Wasser mit dispergierten Hohlglaskugeln mit einem Durchmesser von $10 \mu \mathrm{m}$ eingesetzt. Die Strom-Spannungs-Wandlungen wurden mit zwei FEMTO-Verstärkern DLPCA-200 (Fa. FEMTO Messtechnik GmbH) mit der Verstärkung $10^{6}$ im fotovoltaischen Modus realisiert. Am Oszilloskop (DL750 ScopeCorder, YOKOGAWA) wurde im AC-Modus eine Bandbreite von $5 \mathrm{kHz}$ eingestellt (Signale vgl. Bild 4).

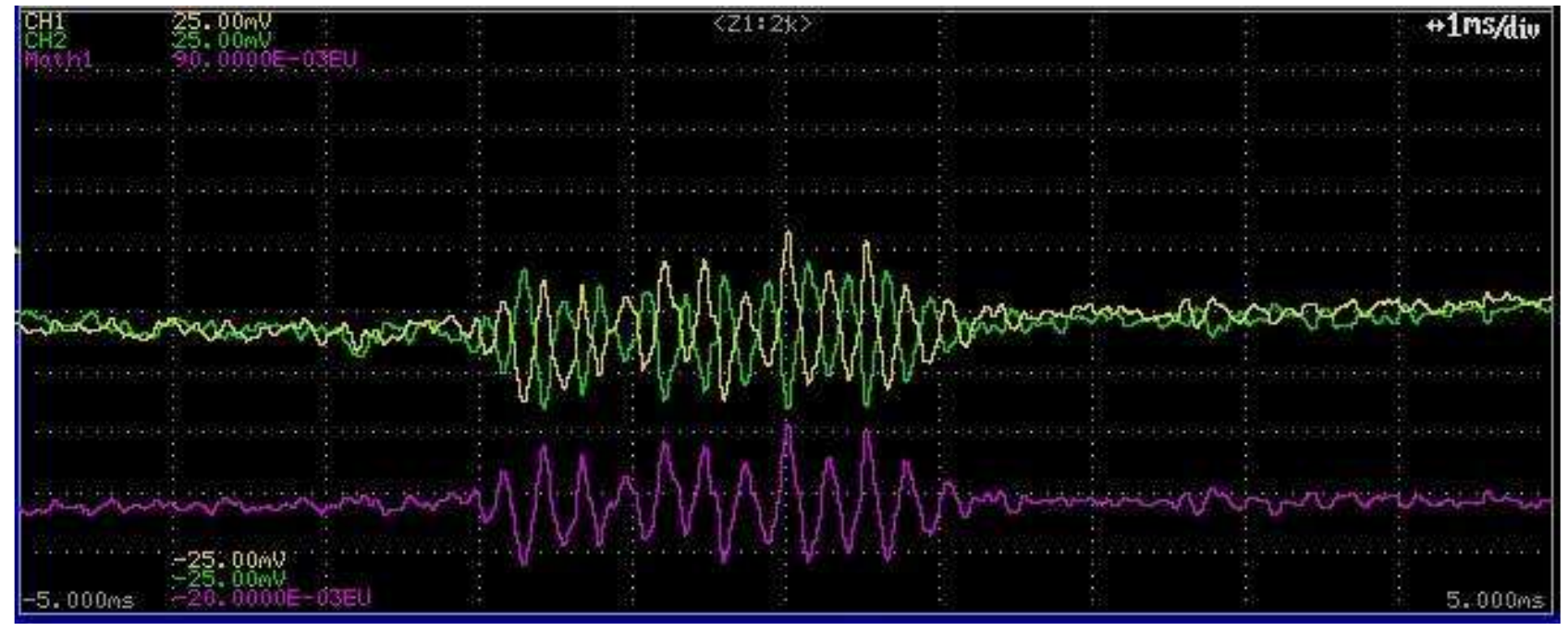

Bild 4 Einzelsignale (oben) und Differenzsignal für einen Si-Chip mit der Gitterkonstante $110 \mu \mathrm{m}$, Partikel: $10 \mu \mathrm{m}$ Hohlglaskugeln, Volumenstrom:20 mL/h.

\section{Literatur}

[1] H. Rauh, J. Zosel, D. Petrak: OPTISCHER SENSOR ZUR STRÖMUNGSMESSUNG IN MIKROSTRUKTUREN. Proc.:Fachtagung "Lasermethoden in der Strömungsmesstechnik", Karlsruhe, 9. - 11. September 2008.

\section{Danksagung}

Die Arbeiten wurden unter dem Förderkennzeichen VF090018 über die EuroNorm GmbH durch das Bundesministerium für Wirtschaft und Technologie innerhalb des Förderprogramms INNO-KOM-OST gefördert. Die Autoren danken für die Unterstützung. Die Autoren bedanken sich auch bei Herrn Prof. Petrak vom Ingenieurbüro FlowSensor GbR für wertvolle Hinweise und die anregenden Diskussionen. 